\title{
Apatite Revisited: The Role of Anisotropy in Apatite Analysis
}

\author{
D.T. Adams, ${ }^{*}$ A.E. Koenig, ${ }^{*}$ H.A. Lowers* \\ * Central Mineral and Environmental Resources Science Center, U.S. Geological Survey, Denver \\ Federal Center - MS973, Denver, CO 80225
}

Damage due to specimen-beam interaction is a well known phenomenon in many different materials including glasses and certain minerals. Apatite is one such mineral found in geological and biological specimens, but its behavior under a focused electron beam is notoriously problematic [1] [2]. One example of the effects of electron bombardment intensity in apatite is the change in fluorine X-ray intensity. Regardless of orientation, F X-ray intensity initially increases then decreases during the analysis. However, the rate of this change is dependent on crystallographic orientation as well as operating conditions. Time dependent intensity (TDI) effects are not isolated to $\mathrm{F}$. Changes in X-ray intensity are also observed in $\mathrm{Ca}, \mathrm{P}, \mathrm{Na}, \mathrm{S}$, and $\mathrm{Cl}$ in apatite. Clear concise methods to obtain accurate electron beam analyses (EDS and WDS) particularly with respect to long analysis time, fine spatial resolution, and relatively high beam currents necessary to obtain low detection limits are lacking. Advances in correction techniques in modern day electron microprobe software and electron microbeam techniques can help in correcting the change in intensity of X-rays during a relatively long WDS analysis and for knowing the precise crystallographic orientation of the apatite grain(s) with respect to the electron beam (e.g. EBSD analysis).

This study examines, comprehensively, the anisotropy of the famous Durango, Mexico apatite standard and the damaging effects of an electron microbeam with respect to crystallographic orientation. The data collected allow improved quantification of acceptable conditions for electron beam analysis of apatite in any orientation. Long count time ( $\geq 3500 \mathrm{~s})$, TDI change analyses were conducted on large $(>1 \mathrm{~cm})$ euhedral Durango Apatite crystals cut parallel and perpendicular to the c-crystallographic axis. The analyses were conducted on a JEOL JSM-8900 electron microprobe using a $15 \mathrm{kV}$ accelerating voltage and beam currents ranging from 4 to $50 \mathrm{nA}$ and spot sizes ranging from 1 to $20 \mu \mathrm{m}$. Analyses were performed using both a multi-layered pseudocrystal LDE1 and standard TAP crystals. Fig. 1 is a plot of the change in count intensity for F versus time for one set of analysis conditions (15 nA) on the LDE1 crystal.

X-ray intensity versus time varies dramatically as a function of spot size, beam current, and crystallographic orientation. These changes, depending on analysis parameters, can be accounted for by using software TDI correction curves. However, if extreme analysis parameters (e.g. $1 \mu \mathrm{m}$ spot size, $50 \mathrm{nA}$ beam current) are used when analyzing apatite parallel or nearly parallel to the C-axis, a TDI software correction cannot correct for the X-ray intensity changes (Fig. 1b). Results in this study are similar to those in Stormer [2], but extend those findings to intensity of $\mathrm{Ca}, \mathrm{P}, \mathrm{Na}, \mathrm{S}$, and $\mathrm{Cl}$ in addition to $\mathrm{F}$. Analyses using other methods, such as laser ablation inductively couple plasma mass spectrometry, are beginning to demonstrate anisotropy effects associated with those analytical protocols.

References

[1] J.C. Stormer, Jr. et al., Am. Mineral. 78 (1993) 641-648.

[2] J.C. Stormer, Jr. et al., Geol. Soc. Am. Abstracts with Programs 23 (1991) A22O 

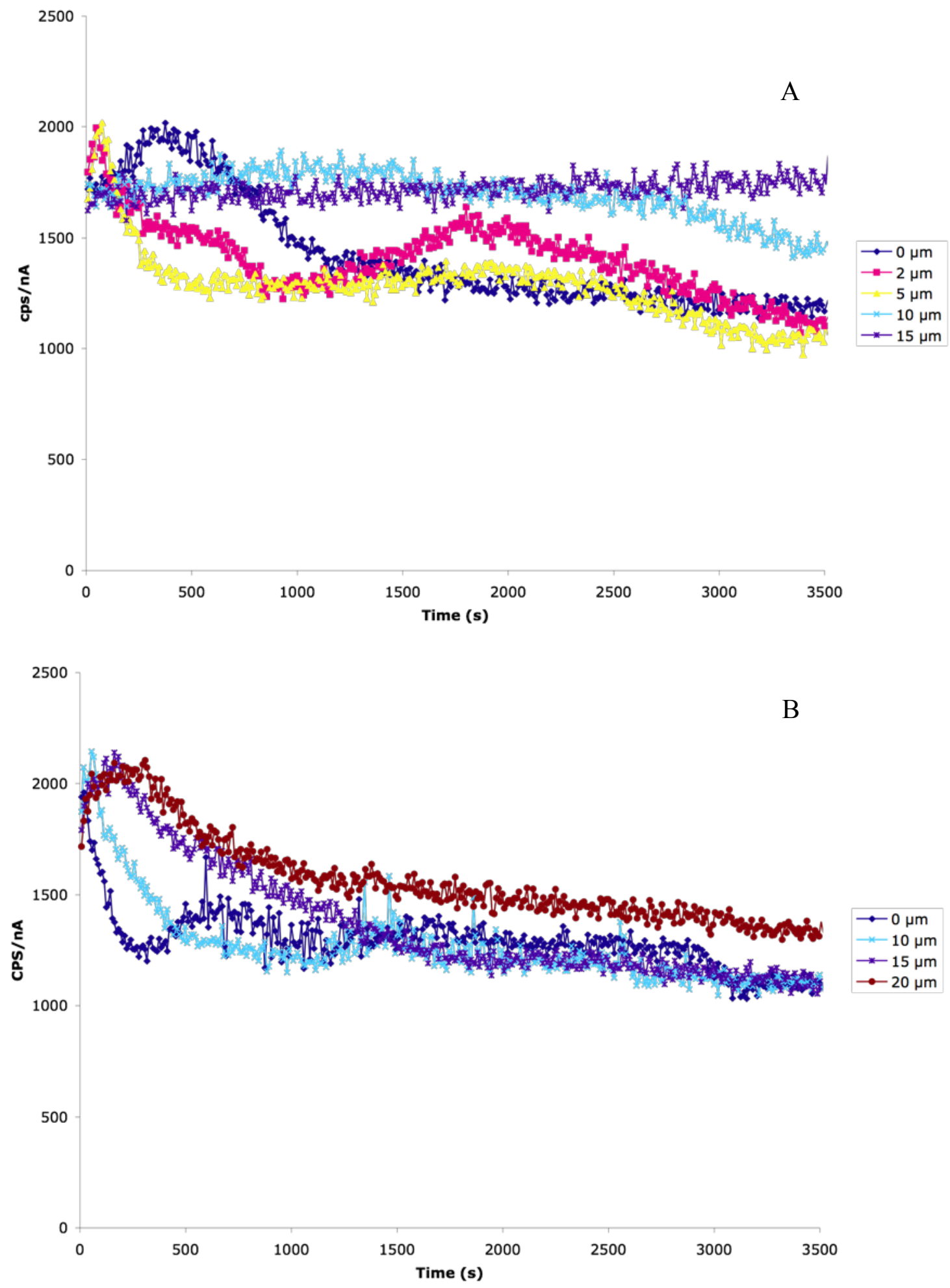

FIG. 1. Time dependent intensity (TDI) change curves for the Durango Apatite during long analysis. Counts recorded every 8.5 seconds; $15 \mathrm{nA}$ beam current. (a) TDI curve for varying spot sizes; electron beam perpendicular to the C-axis. (b) TDI curve for varying sizes; electron beam parallel to the C-axis. 